Russell J. Stewart $\cdot$ Jessica Semerjian Christoph F. Schmidt

\title{
Highly processive motility is not a general feature of the kinesins
}

Received: 16 September 1997 / Accepted: 4 November 1997

\begin{abstract}
Evidence is presented that the kinesin-related ncd protein is not as processive as kinesin. In low surface density motility experiments, a dimeric ncd fusion protein behaved mechanistically more similar to non-processive myosins than to the highly processive kinesin. First, there was a critical microtubule length for motility; only microtubules longer than this critical length moved in low density ncd surfaces, which suggested that multiple ncd proteins must cooperate to move microtubules in the surface assay. Under similar conditions, native kinesin demonstrated no critical microtubule length, consistent with the behavior of a highly processive motor. Second, addition of methylcellulose to decrease microtubule diffusion decreased the critical microtubule length for motility. Also, the rates of microtubule motility were microtubule length dependent in methylcellulose; short microtubules, that interacted with fewer ncd proteins, moved more slowly than long microtubules that interacted with more ncd proteins. In contrast, short microtubules, that interacted with one or a few kinesin proteins, moved on average slightly faster than long microtubules that interacted with multiple kinesins. We conclude that a degree of processivity as high as that of kinesin, where a single dimer can move over distances on the order of one micrometer, may not be a general mechanistic feature of the kinesin superfamily.
\end{abstract}

Key words ncd protein - Kinesins · Processivity · Microtubules · In vitro motility

Abbreviations IPTG Isopropyl-thiogalactoside $\cdot A M P P N P$ 5 '-adenylyl imidodiphosphate $\cdot D T T$ Dithiothreitol $\cdot E G T A$

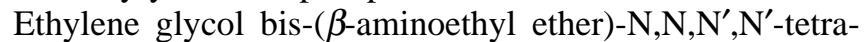

R. J. Stewart (两)

Department of Bioengineering, University of Utah, Salt Lake City, UT 84112, USA

e-mail: rstewart@ee.utah.edu

C. F. Schmidt

Biophysical Research Division, University of Michigan, Ann Arbor, MI 48109, USA

e-mail: cfs@umich.edu acetic acid $\cdot n c d$ Non-claret disjunctional $\cdot$ PIPES 1,4-piperazinediethanesulfonic acid · GST Glutathione S-transferase . DIC Differential interference contrast

\section{Introduction}

The kinesins are a family of motor proteins responsible for intracellular active transport on the microtubule cytoskeleton. A unique kinesin, or kinesins have been found to be associated with most of the moving components of eukaryotic cells (for a recent review see (Moore and Endow 1996)). The kinesin family contains members that move toward the microtubule plus-end and members that move toward the microtubule minus-end, for example the ncd and kar3 proteins (McDonald and Goldstein 1990; Walker et al. 1990; Endow et al. 1994). Therefore, the kinesin family can provide both anterograde and retrograde forces in the cell. The original kinesin, discovered in squid giant axons (Vale et al. 1985), drives anterograde transport of a subset of neuronal vesicles (Gho et al. 1992). Kinesin proteins bound to the surface of vesicles drag the vesicles along axonal microtubules for distances up to a meter. The geometry of a spherical vesicle interacting with an axonal microtubule suggests that perhaps only a few kinesins can interact at one time with the microtubule during vesicle transport. Kinesin, therefore, is likely to work in small numbers, or even alone, to transport its cargo relatively long distances.

Consistent with its biological role of long-distance vesicle transport, dimeric kinesin in vitro is highly processive - it can remain associated with its microtubule track while moving several micrometers, or through hundreds of ATP hydrolysis cycles. The highly processive character of kinesin was first observed in microtubule motility experiments with kinesin immobilized on glass coverslips (Howard et al. 1989). Kinesin surface densities as low as a few molecules per $\mu \mathrm{m}^{2}$ resulted in appreciable rates of microtubule surface association and motility. At low kinesin densities, microtubules pivoted as they moved across 
a stationary point - a behavior referred to as nodal point pivoting. The microtubules, thought to be pivoting on a single kinesin molecule, frequently moved completely to their end before dissociating from the pivot point. Motility experiments with low densities of kinesin on silica beads confirmed that single kinesins were capable of moving several micrometers (Block et al. 1990). Experiments with sufficient resolution to observe single consecutive steps of a single dimeric kinesin moving a bead provided direct evidence of processive motion (Svoboda et al. 1993; Coppin et al. 1996). Kinetic analysis of ATP hydrolysis by kinesin in solution (Gilbert et al. 1995; Hackney 1995), and observations of motility of single, fluorescently-labeled kinesin in solution (Vale et al. 1996) provided additional evidence of kinesin's high degree of processivity.

Models for the molecular basis of highly processive motility by single dimeric kinesin molecules invoke handover-hand mechanisms that keep alternating motor domains tightly bound to the microtubule as the motor moves along the microtubule surface (Howard et al. 1989; Hackney 1994). In hand-over-hand motility models, the microtubule-bound motor domain is prevented from dissociating from the microtubule until the unattached motor domain has entered, or has a high probability of entering a strong binding state one subunit further along on the microtubule surface. Consistent with hand-over-hand models, kinesin's paired motor domain structure is important for high processivity. Single molecules of dimeric native kinesin followed a non-random path parallel to the axis of the protofilaments for distances of several micrometers (Ray et al. 1993). The non-random path was a further manifestation of kinesin's processive mechanism that kept the motor bound to the microtubule between translocation events and thereby determined the path of the motor. In contrast, beads densely coated with single-headed kinesin staggered along a random path on the microtubule surface, suggesting that single kinesin domains rebound the microtubule randomly after release from the microtubule between power strokes (Berliner et al. 1995).

Among cytoskeletal motor proteins, processivity to a degree that generates hundreds of coordinated ATP hydrolysis and translocation events is, so far, unique to the original kinesin. Neither the myosins nor the dyneins that have been studied in vitro demonstrated highly processive motility (Toyoshima et al. 1990; Wang et al. 1995). Many members of these families, though not all, work together in large arrays - myosin in muscle fibers and dynein in flagellar axonemes. Their biological functions offer a rationalization for their non-processivity; motors strongly bound between power strokes would constrain other motors trying to move the same substrate. Among other members of the kinesin family, including the ncd protein, mechanistic studies have not addressed processivity. It is reasonable to expect, though, that kinesins with transport functions similar to the original kinesin - functions that imply small numbers of independent motors - will be highly processive. On the other hand, kinesins that work in microtubule bundles, for example kinesins that function in spindle morphogenesis or operation, may be much less processive or non-processive. The later category may include the ncd protein, which functions in proper meiotic spindle assembly in female Drosophila, possibly by crosslinking microtubules at the spindle poles (Kimble and Church 1983; Hatsumi and Endow 1992). Consistent with its cellular role, purified ncd protein bundled microtubules in vitro (McDonald and Goldstein 1990).

To address whether a dimeric ncd motor protein shares kinesin's highly processive character, microtubule motility experiments were done with relatively low surface densities of the ncd protein and native kinesin under identical conditions. We found that an ncd fusion protein containing the motor domain and the entire predicted coiled-coil stalk, shown previously to be dimeric (Chandra et al. 1993), was not highly processive.

\section{Experimental procedures}

\section{Construction of pGEX-N195}

Construction of pGEX-N195 was described previously (Stewart et al. 1993). The fusion protein encoded by pGEX-N195 contains glutathione S-transferase, the pGEX-2T thrombin cleavage site, the sequence GPI from the polylinker, and amino acids 195 to 685 of ncd. This fusion protein, referred to as GST-N195, is similar to the GST-MC1 fusion protein described by Chandra and collaborators (Chandra et al. 1993). The GST-MC1 protein contains GST fused to amino acid 210 of ncd. The discrepancy in the numbering of amino acids is due to the choice of the start codon in the cDNA. In our numbering scheme, using the second ATG in the ncd cDNA as the initiator codon, amino acid 195 is the lysine residue encoded by the unique Afl II restriction site of ncd. In the numbering scheme used by Chandra (Chandra et al. 1993), which uses the first ATG in the ncd cDNA as the initiator, amino acid 210 is the lysine residue encoded by the unique Afl II site.

\section{Purification of motor proteins and microtubules}

GST-N195 was affinity purified from E. coli using glutathione-agarose, as described previously (Stewart et al. 1993). Briefly, GST-N195 expression was induced by the addition of $0.1 \mathrm{mM}$ IPTG $^{3}$ to approximately mid-log cultures of E. coli, which were then shaken at $20-22^{\circ} \mathrm{C}$ for additional 4-6 hours. After harvesting the cells by centrifugation, they were resuspended in lysis buffer (20 mM PO ${ }_{4}, 1 \mathrm{~mm} \mathrm{ETD,} 150 \mathrm{~mm} \mathrm{NaCl}, \mathrm{pH}$ 7.2), lysed by sonication. The lysate was clarified by centrifugation (17,000 rpm, Beckman JA-17 rotor). Glutathione-agarose beads (Sigma) were added to the lysate. After mixing at $4^{\circ} \mathrm{C}$ for $30-60$ minutes, the beads were washed extensively with PEM80 buffer ( $80 \mathrm{~mm}$ Pipes, $1 \mathrm{mM}$ EGTA, $4 \mathrm{~mm}$ $\mathrm{Mg}^{2+}, \mathrm{pH}$ 6.9). GST-N195 was then eluted with $10 \mathrm{~mm}$ glutathione in PEM80. GST-N195 was frozen in liquid $\mathrm{N}_{2}$ in small aliquots and stored at $-80^{\circ} \mathrm{C}$. Kinesin was purified 
from squid optic lobes as described previously (Vale et al. 1985) and stored at $-20^{\circ} \mathrm{C}$ in $50 \%$ glycerol. Tubulin was purified from bovine brain following standard recipes (Williams and Lee 1982) and stored at $-80^{\circ} \mathrm{C}$.

\section{Microtubule binding and motility assays}

Motility assays were done in flowchambers assembled with two coverslips and narrow strips of double-stick tape (Scotchbrand, 3M). The double coverslip flowchambers were attached to a stainless steel holder for mounting on the microscope stage. For native kinesin motility assays, coverslips were prepared by cleaning in a solution of saturated $\mathrm{KOH} / \mathrm{ethanol}$ at room temperature, followed by extensive rinsing with distilled water. For GST-N195 motility assays, $\mathrm{KOH} /$ ethanol cleaned coverslips were silanized by dipping in a solution of $10 \%$ (v/v) Sigmacote (Sigma) in heptane. The Sigmacote surfaces were found to give the most reliable motility with GST-N195.

Low surface density motility assays were done by diluting kinesin or GST-N195 into assay buffer $(80 \mathrm{~mm}$ $\mathrm{K}_{2}$-Pipes, $1 \mathrm{~mm}$ EGTA, $4 \mathrm{~mm} \mathrm{Mg}{ }^{2+}, 1 \mathrm{~mm}$ DTT, $1 \mathrm{mg} / \mathrm{ml}$ casein, $20 \mu \mathrm{M}$ taxol). The diluted motor proteins were added to flowchambers (approximately $10 \mu$ l volume) preloaded with assay buffer. After 5-10 minutes, the flowchamber was washed with 50-100 $\mu$ l assay buffer. Microtubules, diluted appropriately in assay buffer plus $2.5 \mathrm{mM}$ MgATP or $2.5 \mathrm{mM}$ MgAMPPNP, were added to the flowchamber. Binding and motility of microtubules on the flowchamber surface were observed by video-enhanced DIC microscopy, and recorded on videotape.

The lengths of bound microtubules were measured with a ruler from video prints (Sony, UP-880) of the video recorded assays. The magnification of the microscope was calibrated with a micrometer-scale slide. Likewise, motility rates were determined by measuring the distance a microtubule moved between video prints at defined time intervals.

\section{Results}

Microtubule motility with low GST-N195 surface densities

GST-N195 $(0.5 \mu \mathrm{g}$ in $10 \mu \mathrm{l}$ assay buffer $)$ was added to a $3 \times 20 \mathrm{~mm}$ flow chamber assembled with Sigmacote silanized coverslips. When microtubules and $2.5 \mathrm{mM} \mathrm{MgATP}$ were added to the flowchamber, large numbers of microtubules moved over the surface of the flowchamber. Long microtubules (greater than $10 \mu \mathrm{m}$ ) moved across the entire field of view; shorter microtubules (less than $10 \mu \mathrm{m}$ ) moved, but frequently dissociated from the surface. Assuming that all of the GST-N195 bound to the flowchamber surfaces and remained bound after washing the flowchamber, the maximal GST-N195 surface density in this assay was about 16,000 dimeric molecules $\mu \mathrm{m}^{-2}$. This number may not have been an accurate estimate of the density of functional motors on the surface, since the fraction of motors that bound, remained bound after washing the flowchamber, and that remained active after binding was not known. Because we were interested in relative differences, we did not attempt to determine the absolute density of functional motors. References to surface densities are estimated maximum densities. With a nominal motor surface density of $4000 \mu \mathrm{m}^{-2}-0.125 \mu \mathrm{g}$ GST-N195 added to an equivalent flow chamber - microtubules added with $2.5 \mathrm{~mm}$ MgATP did not associate, even transiently, with the flowchamber surface.

Possible explanations for the absence of GST-N195 motility at comparatively high surface densities were: GSTN195 adsorbed slowly or weakly to the flowchamber surface, GST-N195 was inactivated as a result of adsorption to the surface, or there was a critical GST-N195 surface density necessary for microtubule motility, or a combination of these factors. These possibilities were tested by adding the non-hydrolyzable ATP analog, AMPPNP, to the flowchamber. Since GST-N195, like kinesin, sedimented with microtubules in the presence of AMPPNP, it seemed reasonable to expect that even single molecules of active GST-N195 on the flowchamber surface would bind microtubules in the presence of AMPPNP. When ATP was washed out and microtubules were added to the flowchamber with $2.5 \mathrm{mM}$ MgAMPPNP, microtubules bound immediately to the surface. Short microtubules (less than $2 \mu \mathrm{m}$ ) frequently pivoted around a single point briefly before becoming completely immobilized. When $2.5 \mathrm{~mm} \mathrm{MgATP}$ was added back to the flowchamber, the bound microtubules completely dissociated from the surface within seconds. These observations demonstrated that active GST-N195, capable of reversible, nucleotide-dependent microtubule binding, was present on the flowchamber surface. Therefore, a critical surface density of GST-N195 was required for microtubule motility; a behavior quite distinct from native kinesin.

Length distributions of microtubules bound by GST-N195 in AMPPNP and ATP

The lengths of microtubules bound in MgATP and MgAMPPNP were determined at a GST-N195 surface density that supported microtubule motility in MgATP, nominally $15,000 \mu^{-2}$. The length distribution of microtubules bound and moving on the flowchamber surface in MgATP is shown in Fig. $1 \mathrm{a}$. Microtubules less than $4 \mu \mathrm{m}$ long were only 9 percent of the total number of bound microtubules. Short microtubules associated only transiently with the surface in the presence of MgATP (Fig. 1b), and were never conclusively observed to bind and pivot around a single point. When the same solution of microtubules was added with MgAMPPNP to the same GST-N195 flow chamber, microtubules that bound to the flowchamber surface had a significantly different length distribution which was more typical of microtubules reassembled in vitro, that is, skewed toward short lengths (Fig. 1c). Microtubules less than $4 \mu \mathrm{m}$ long were well represented in the 

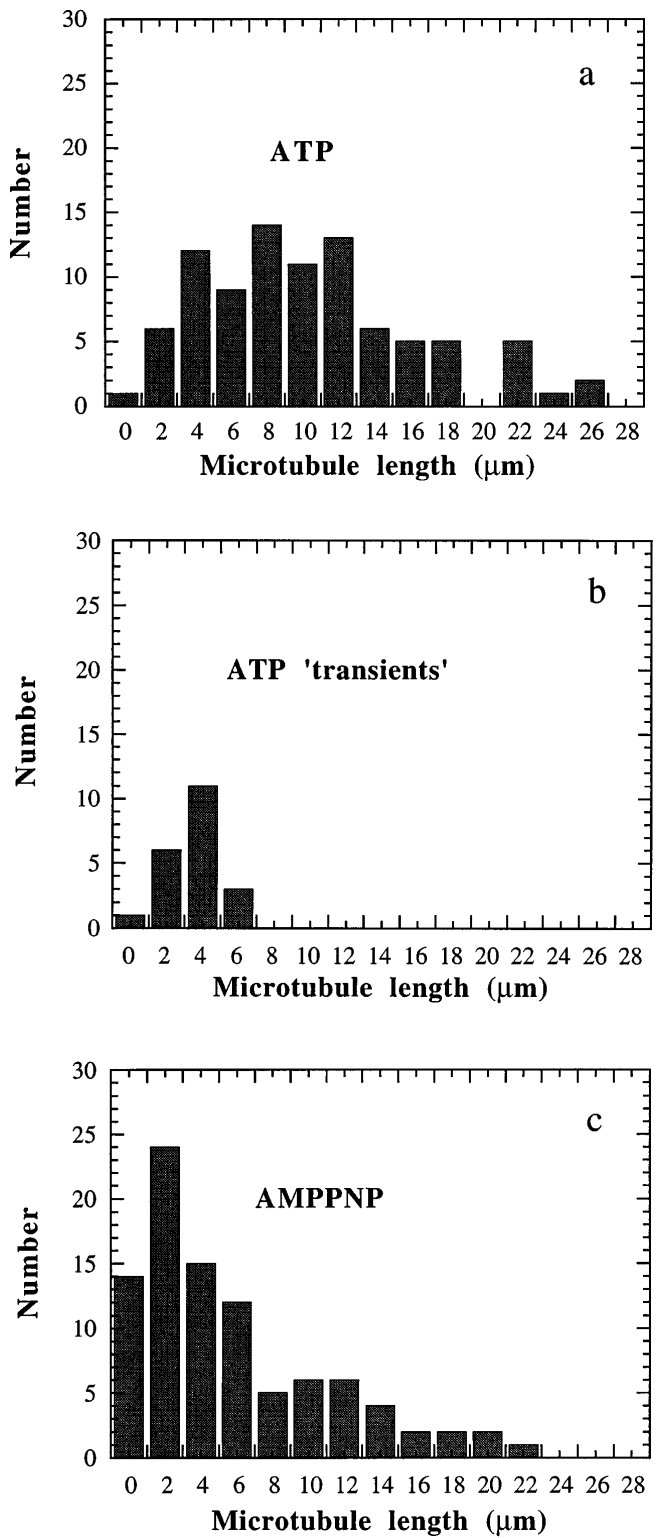

Fig. 1 a-c Length distributions of microtubules bound by GSTN195 at a relatively high surface density (nominally $15,000 \mu^{-2}$ ). a Lengths of microtubules bound in $2.5 \mathrm{mM} \mathrm{Mg}$ ATP. Average length was $11.7 \mu \mathrm{m}, n=90$. b Lengths of microtubules bound transiently to the flowchamber surface in $2.5 \mathrm{~mm} \mathrm{MgATP}$. Average length was $4.5 \mu \mathrm{m}, n=25$. $\mathrm{c}$ Lengths of microtubules bound to the flowchamber surface in $2.5 \mathrm{mM} \mathrm{MgAMPPNP}$. The average length was $7.5 \mu \mathrm{m}, n=93$

MgAMPPNP distribution, 45 percent of the total. The MgAMPPNP control experiment demonstrated that active GST-N195 was present on the flowchamber surface, and that the distribution of lengths in the microtubule solution added to the flow chamber contained a high proportion of short microtubules. Therefore, the differences in the microtubule distributions bound in MgATP and MgAMPPNP were due to differences in the effective affinity of surface bound GST-N195 for microtubules in MgATP versus MgAMPPNP. The $\mathrm{P}$ value of a $\chi^{2}$ significance test of the difference between the two histogrammed data sets (Press et al. 1992) was 0.000029 .
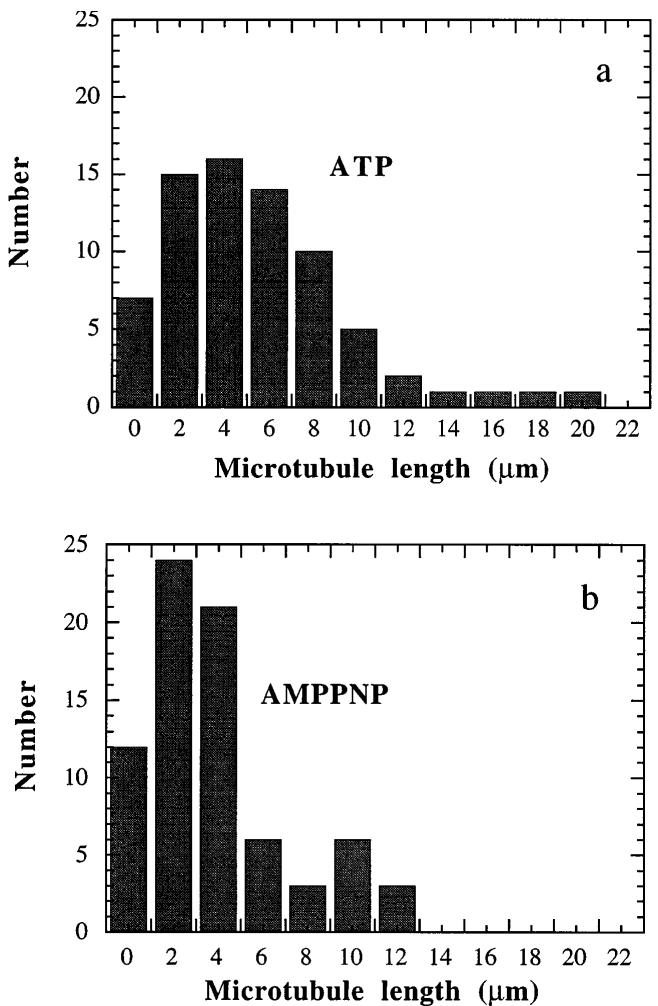

Fig. 2 a, b Length distributions of microtubules bound by native kinesin. a Lengths of microtubules bound to the flowchamber surface in $2.5 \mathrm{~mm} \mathrm{MgATP}$. Average length was $6.6 \mu \mathrm{m}, n=74$. b Lengths of microtubules bound to the flowchamber surface in $2.5 \mathrm{~mm}$ $\operatorname{MgAMPPNP}$. The average length was $4.8 \mu \mathrm{m}, n=76$

Length distributions of microtubules bound by kinesin in AMPPNP and ATP

The lengths of microtubules bound in MgATP or MgAMPPNP by kinesin at low surface densities were measured. The kinesin dilution was determined empirically to give reasonable microtubule association rates and frequent nodal point pivoting of short microtubules, but the absolute kinesin surface density was not estimated. In MgATP, the lengths of bound microtubules had a distribution that was similar to the distribution of a typical microtubule population (Fig. 2a). Microtubules less than $4 \mu \mathrm{m}$ long bound in the presence of MgATP were 56 percent of the total. In MgAMPPNP, the distribution of bound microtubules was similar to the distribution in $\operatorname{MgATP}$ (Fig. 2b). Microtubules less than $4 \mu \mathrm{m}$ long were 40 percent of the total population. The microtubule preparation used for the kinesin experiments was gently sheared before use so that it contained more short microtubules, and therefore had a narrower distribution and a shorter mean length than the microtubule preparation used for the GST-N195 experiments. The $\mathrm{P}$ value of a $\chi^{2}$ test of the difference between the two histogrammed data sets was 0.11 , suggesting that the difference was not highly significant. The similar length distributions of microtubules bound in MgAMPPNP and microtubules moving in MgATP were consistent with expectations for highly processive kinesin. Since even very 
short microtubules can bind and move in a single kinesin molecule for an observable distance in the presence of MgATP, we expected no threshold microtubule length for microtubule binding.

Microtubule length dependence of GST-N195 motility rates

The critical GST-N195 surface density for microtubule motility we observed was similar to the critical surface density previously observed for actin filament motility driven by muscle myosin (Harada et al. 1990; Toyoshima et al. 1990). By adding methylcellulose to the motility assay, actin filament motility was observed below the critical myosin density (Uyeda et al. 1990). The limited lateral diffusion of the actin filaments in methylcellulose raised the probability that the filament would remain in the proximity of the motor between power strokes. We added low concentrations of methylcellulose to GST-N195 motility experiments as another way to test for a low degree of processivity. In the absence of motor proteins, microtubule diffusion in $0.2 \%$ methylcellulose was greatly restricted in the direction perpendicular to the long axis of the microtubule, but relatively unimpeded in the parallel direction. Microtubules also tended to be excluded from the methylcellulose solution onto the coverslip surface, and into bundles on the surface and in solution. In methylcellulose, short microtubules skittered around on the surface, intermediate length microtubules diffused predominantly along their long axis, and long microtubules laid relatively motionless on the flowchamber surface.

In a flowchamber with a nominal GST-N195 surface density of $4000 \mu^{-2}$, no motility was observed when microtubules were added with $2.5 \mathrm{~mm} \mathrm{MgATP}$ and without methylcellulose. When microtubules in assay buffer plus $0.2 \%$ methylcellulose were added to the same flowchamber, long microtubules (greater than approximately $10 \mu \mathrm{m}$ ) moved smoothly and continuously, while periods of random shuffling interrupted the movement of shorter microtubules. For a non-processive motor the motility rate of short microtubules in methylcellulose was a complex function of microtubule length and surface distribution of motors. The motility rate reflected microtubule movements averaged over periods of motion driven by varying numbers of motors and periods of diffusional motion. Qualitatively, shorter microtubules interacting with fewer motors were expected to spend a smaller fraction of time undergoing directed, motor-driven motion and should have moved at a reduced average rate. The rates of microtubule movement plotted as a function of microtubule length (Fig. 3, closed symbols) trended toward slower rates with shorter microtubules. Comparison of the mean motility rates for microtubule lengths less than the median length of $9 \mu \mathrm{m}(181 \pm 7 \mathrm{~nm} / \mathrm{s} ; n=19)$, and lengths greater than the median length $(203 \pm 5 \mathrm{~nm} / \mathrm{s} ; n=20)$ suggested that the trend was statistically significant. The $\mathrm{P}$ value of a $t$-test for the significance was 0.01 . For comparison, rates of microtubule movement measured in an assay with a nominal

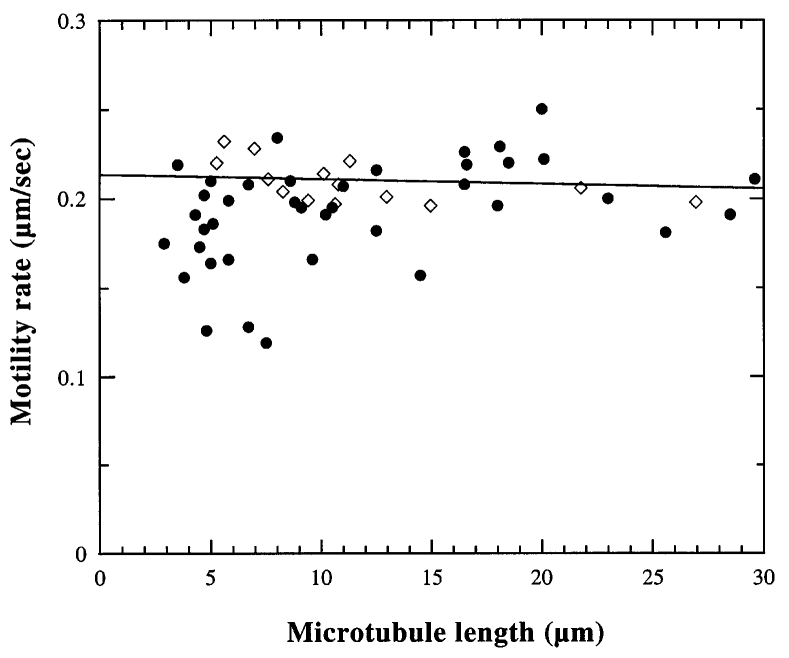

Fig. 3 Microtubule motility rates as a function of microtubule length for GST-N195. Microtubule motility rates were determined in the presence of $0.2 \%$ methylcellulose on a relatively low-density (nominally $4000 \mu^{-2}$ ) GST-N195 surface that did not support microtubule motility without methylcellulose. Motility rates are plotted as a function of the microtubule length (solid symbols). The microtubule length is proportional to the number of motors that could interact simultaneously with the microtubule. Microtubule motility rates for a high surface density GST-N195 (nominally $\geq 60,000 \mu^{-2}$ ) motility experiment are also plotted (open symbols) as a function of microtubule length

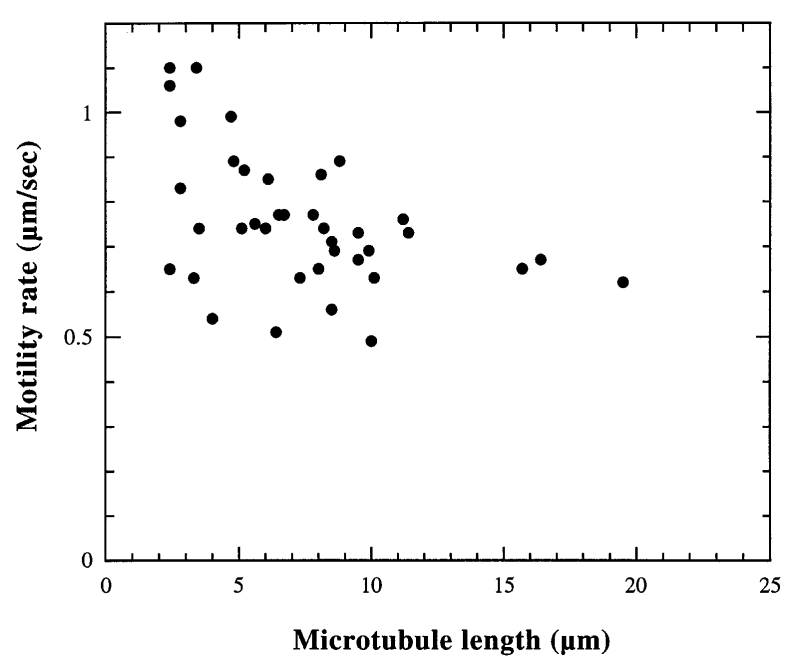

Fig. 4 Microtubule motility rates as a function of microtubule length for native kinesin. Microtubule motility rates were determined on a low density squid kinesin surface and plotted as a function of the microtubule length (solid symbols)

GST-N195 surface density of $60,000 \mu \mathrm{m}^{-2}$, without methylcellulose, showed no dependence on microtubule length (Fig. 3, open symbols).

In contrast, the average rate of microtubule movement in low density kinesin motility experiments, plotted as a function of microtubule length (Fig. 4), appeared to increase for shorter microtubules. Comparison of the mean motility rates for microtubules shorter than the median length of $7 \mu \mathrm{m}(816 \pm 42 \mathrm{~nm} / \mathrm{s} ; n=19)$, and greater than the 
median length $(692 \pm 22 \mathrm{~nm} / \mathrm{s} ; n=19)$ suggested that the apparent trend was statistically significant. The $t$-test $\mathrm{P}$ value was 0.005 .

\section{Discussion}

Highly processive motility is one of the distinguishing features of the mechanism of the original kinesin. Whether highly processive motility is a general feature of the kinesin family, or a feature of a subset of family members with similar transport functions has not been experimentally addressed. This question is of particular interest for minusend motors, like the ncd protein, since additional mechanistic information may provide clues as to what determines the direction that kinesin motors move.

\section{Structure of GST-N195}

Two recent reports (Case et al. 1997; Henningsen and Schliwa 1997) have shown that a plus-end directed ncd motor domain can be constructed by splicing the kinesin neck region and stalk onto the 320 amino acid catalytic domain of ncd. The GST-N195 protein used in our experiments contains the entire ncd motor domain, including the neck region, as well as the entire predicted coiled-coil stalk. On that basis, we expect GST-N195 to be dimeric and behave similarly to native ncd. Because evidence suggests that the dimeric structure of kinesin is important for processivity (Berliner et al. 1995), we were concerned about the structure of GST fusion proteins. Glutathione S-transferase from $S$. japonicum - the GST gene used in the pGEX vector (Smith and Johnson 1988) - is a dimer (McTigue et al. 1995). It was possible that dimerization of the GST portion of the fusion protein interfered with the normal dimerization of the ncd portion, perhaps disrupting or interfering with a linkage between subunits potentially responsible for processivity. This doesn't seem likely, though, since in the crystal structure the carboxy termini of the GST monomers are exposed on the same side of the GST dimer and are separated by approximately 40 angstroms (McTigue et al. 1995). The flexible eleven amino acid peptide (SDLVPRGSPGI) separating the final residue of GST and amino acid 195 of ncd should allow normal dimerization of ncd. The dumb-bell shaped molecules observed in electron micrographs of a rotary shadowed ncd-GST fusion equivalent to GST-N195 (Chandra et al. 1993) most likely represented a globular GST dimer separated by the ncd coiled-coil stalk from a pair of ncd motor domains.

\section{Processivity of motor proteins}

A motor protein is generally classified as processive when it remains associated with its track through multiple enzymatic cycles. A degree of processivity can be defined as the probability that an individual motor will remain associated with its track through one enzymatic cycle. This probability could be high when the two heads of a dimer, for example, bind strongly in a coordinated hand-over-hand mechanism. Weak coordination, or weak intermediate binding states could lead to a diminished degree of processivity, that is, on average a smaller number of consecutive steps. Processivity extremes are represented by kinesin and myosin, both of which have been investigated in experiments with single translocation resolution. Single dimeric kinesin molecules were capable of processive movement against forces, applied with an optical trap, for distances of at least $200 \mathrm{~nm}$ (the radius of an optical trap) and forces up to 5-6 piconewtons (Svoboda et al. 1993; Meyhöfer and Howard 1995; Coppin et al. 1996; Gittes et al. 1996). These experiments directly demonstrated that there is a strong microtubule binding state between translocation events, capable of supporting relatively high forces, that is part of kinesin's processive mechanism. Myosin, on the other hand, is non-processive; in experiments capable of resolving individual translocation and force generation events there was no evidence of correlated translocations by single dimeric molecules of skeletal muscle heavy meromyosin (Finer et al. 1994; Molloy et al. 1995). These results showed that myosin is non-processive partly because it enters a weak binding state between translocation events that is not capable of supporting even small forces.

\section{Processivity of GST-N195}

In prior motility experiments with native kinesin, the attachment rate of microtubules to the surface decreased gradually as the kinesin surface density decreased, provided that surface denaturation of kinesin was prevented (Howard et al. 1989). Densities as low as a few kinesins per $\mu \mathrm{m}^{2}$ resulted in appreciable attachment rates and microtubule movement. In similar experiments with GST-N195, we observed no microtubule attachment or motility below a threshold surface density, although active GST-N195 was present on the surface as indicated by reversible microtubule binding in the presence of MgAMPPNP. Above the threshold density, microtubule attachment and motility showed a distinct length dependence (Fig. 1). The threshold surface density and microtubule length dependence of motility suggested that there was a critical number of GST-N195 motors necessary to ensure that at least one motor was in a strong binding state at all times. Only microtubules long enough to interact with the critical number of motors attached to the surface and moved for an observable period of time. In control experiments, using the same reagents and conditions, attachment and movement was independent of microtubule length at low native kinesin surface densities (Fig. 2), as expected for a highly processive motor. The threshold length for microtubule movement at low GST-N195 surface densities suggested that the critical number for GST-N195 was considerably greater than the critical number for native kinesin (one). Because the binding efficiency of GST-N195 to the flowchamber surface was unknown, we could not accurately estimate the critical number for GST-N195. 
Motility experiments using methylcellulose to reduce microtubule diffusion confirmed the lower degree of processivity of GST-N195. Microtubules moved smoothly and continuously in the presence of methylcellulose at GSTN195 surface densities that did not support microtubule movements without methylcellulose. Consistent with expectations that for a non-processive motor the motility rates of multiple motors are additive up to the threshold number of motors, we found that the rates of microtubule movement in methylcellulose were microtubule length dependent at GST-N195 surfaces densities below the threshold density; short microtubules moved more slowly than longer microtubules (Fig. 3). This implied that individual GST-N195 motors spent a substantial part of their enzymatic cycle in a weak-binding, non-force generating state. Similar phenomena, a threshold surface density and actin filament length-dependent motility rates, have been reported for muscle myosin (Toyoshima et al. 1990; Uyeda et al. 1990). In control experiments with native kinesin under the same conditions, we found that short microtubules interacting with one or a few kinesins appeared to move slightly faster than long microtubules interacting with several or many kinesins (Fig. 4).

The direct comparisons in our experiments of GSTN195 and native kinesin suggested that GST-N195 was much less processive than the original kinesin, and in standard motility experiments behaved more similarly to nonprocessive myosin. A similar conclusion was reached by Case et al. (1997), who were unable to observe fluorescently labeled ncd proteins, free in solution, bind and move measurable distances on surface-immobilized microtubules. In contrast, free fluorescently labeled kinesin proteins moved easily observable distances; on average, a micrometer. Neither our experiments, nor the experiments of Case et al., had sufficient spatial and temporal resolution to conclude that ncd is non-processive like myosin. It remains possible that ncd is processive in a mechanistically similar way to kinesin, but that the microtubule binding state between translocations is much weaker, resulting in many fewer concerted steps. Whether ncd is weakly processive or non-processive will be resolved ultimately by high-resolution, single-molecule motility experiments similar to the experiments done with kinesin and myosin. The results presented here will help guide the design of these single-molecule experiments.

Acknowledgements We gratefully acknowledge support by the Rowland Institute for Science, Cambridge, MA, and by the National Science Foundation (grant BIR 95-12699). We thank Frederick Gittes for helpful discussions.

\section{References}

Berliner E, Young EC, Anderson K, Mahtani HK, Gelles J (1995) Failure of a single-headed kinesin to track parallel to microtubule protofilaments. Nature 373:718-721

Block SM, Goldstein LS, Schnapp BJ (1990) Bead movement by single kinesin molecules studied with optical tweezers. Nature 348:348-352
Case RB, Pierce DW, HomBooher N, Hart CL, Vale RD (1997) The directional preference of kinesin motors is specified by an element outside of the motor catalytic domain. Cell 90:959-966

Chandra R, Salmon ED, Erickson HP, Lockhart A, Endow SA (1993) Structural and fucntional domains of the Drosophila ncd microtubule motor protein. J Biol Chem 268:9005-9013

Choppin CM, Finer JT, Spudich JA, Vale RD (1996) Detection of sub-8-nm movements of kinesin by high-resolution optical-trap microscopy. Proc Natl Acad Sci USA 93: 1913-1917

Endow Sa, Kang SJ, Satterwhite LL, Rose MD, Skeen VP, Salmon ED (1994) Yeast Kar3 is a minus-end microtubule motor protein that destabilizes microtubules preferentially at the minus ends. EMBO J 13:2708-2713

Finer JT, Simmons RM, Spudich JA (1994) Single myosin molecule mechanics: piconewton forces and nanometre steps. Nature 368: 113-119

Gho M, McDonald K, Ganetzky B, Saxton WM (1992) Effects of kinesin mutations on neuronal functions. Science 258:313-316

Gilbert SP, Webb MR, Brune M, Johnson KA (1995) Pathway of processive ATP hydrolysis by kinesin. Nature 373:671-676

Gittes F, Meyhöfer E, Sung B, Howard J (1996) Directional loading of the kinesin motor molecule at it buckles a microtubule. Biophys J 70:418-429

Hackney DD (1994) Evidence for alternating head catalysis by kinesin during microtubule-stimulated ATP hydrolysis. Proc Natl Acad Sci USA 91:6865-6869

Hackney DD (1995) Highly processive microtubule-stimulated ATP hydrolysis by dimeric kinesin head domains. Nature $377: 448-$ 450

Harada Y, Sakurada K, Aoki T, Thomas DD, Yanagida T (1990) Mechanochemical coupling in actomyosin energy transduction studied by in vitro movement assay. J Mol Biol 216:49-68

Hatsumi M, Endow SA (1992) Mutants of the microtubule motor protein, nonclaret disjunctional, affect spindle structure and chromosome movement in meiosis and mitosis. J Cell Sci 101:547-559

Henningsen U, Schliwa M (1997) Reversal in the direction of movement of a molecular motor. Nature 389:93-96

Howard J, Hudspeth AJ, Vale RD (1989) Movement of microtubules by single kinesin molecules. Nature 342:154-158

Kimble M, Church K (1983) Meiosis and early cleavage in Drosophila melanogaster eggs: effects of the claret-non-disjunctional mutation. J Cell Sci 62:301-318

McDonald HB, Goldstein LS (1990) Identification and characterization of a gene encoding a kinesin-like protein in Drosophila. Cell 61:991-1000

McTigue MA, Williams DR, Tainer JA (1995) Crystal structure of a schistosomal drug and vaccine traget: glutathione S-transferase from Schistosoma japonica and its complex with the leading antoschistosomal drug praziquantel. J Mol Biol 246:21-27

Meyhöfer E, Howard J (1995) The force generated by a single kinesin molecule against an elastic load. Proc Natl Acad Sci USA 92:574-578

Molloy JE, Burns JE, Kendrick-Jones J, Tregear RT, White DC (1995) Movement and force produced by a single myosin head. Nature 378:209-212

Moore JD, Endow SA (1996) Kinesin proteins: a phylum of motors for microtubule-based motility. Bioessays 18:207-219

Press WH, Flannery BP, Teukolsky SA, Vetterling WT (1992) Numerical recipes in C: the art of scientific computing, 2nd edn. Cambridge University Press, Cambridge, New York

Ray S, Meyhöfer E, Milligan RA, Howard J (1993) Kinesin follows the microtubule's protofilament axis. J Cell Biol 121:1083-1093

Smith DB, Johnson KS (1988) Single-step purification of polypeptides expressed in Escherichia coli as fusion with glutathione S-transferase. Gene 67:31-40

Stewart RJ, Thaler JP, Goldstein LS (1993) Direction of microtubule movement is an intrinsic property of the motor domains of kinesin heavy chain and Drosophila ncd protein. Proc Natl Acad Sci USA 90:5209-5213

Svoboda K, Schmidt CF, Schnapp BJ, Block SM (1993) Direct observation of kinesin stepping by optical trapping interferometry. Nature 365:721-727 
Toyoshima YY, Kron SJ, Spudich JA (1990) The myosin step size: measurement of the unit displacement per ATP hydrolyzed in an in vitro assay. Proc Natl Acad Sci USA 87:7130-7134

Uyeda TQ, Kron SJ, Spudich JA (1990) Myosin step size. Estimation from slow sliding movement of actin over low densities of heavy meromyosin. J Mol Biol 214:699-710

Vale RD, Funatsu T, Pierce DW, Romberg L, Harada Y, Yanagida T (1996) Direct observation of single kinesin molecules moving along microtubules. Nature 380:451-453

Vale RD, Reese TS, Sheetz MP (1985) Identification of a novel forcegenerating protein, kinesin, involved in microtubule-based motility. Cell 42:39-50
Walker RA, Salmon ED, Endow SA (1990) The Drosophila claret segregation protein is a minus-end directed motor molecule [see comments]. Nature 347:780-782

Wang Z, Khan S, Sheetz MP (1995) Single cytoplasmic dynein molecule movements: characterization and comparison with kinesin. Biophys J 69:2011-2023

Williams Jr. RC, Lee JC (1982) Preparation of tubulin from brain. In: Frederiksen DW, Cunningham LW (eds) Structural and Contractile Proteins (Part B: The contractile apparatus and the cytoskeleton). Methods in Enzymology, vol 85. Academic Press, San Diego, pp 376-385 